\title{
Cervicovaginal Bacteriology and Antibiotic Sensitivity Patterns among Women with Premature Rupture of Membranes in Mulago Hospital, Kampala, Uganda: A Cross-Sectional Study
}

\author{
Milton W. Musaba, ${ }^{1}$ Mike N. Kagawa, ${ }^{2}$ Charles Kiggundu, ${ }^{3}$ \\ Paul Kiondo, ${ }^{2}$ and Julius Wandabwa ${ }^{4}$ \\ ${ }^{1}$ Mbale Regional Referral \& Teaching Hospital, P.O. Box 921, Mbale, Uganda \\ ${ }^{2}$ School of Medicine, College of Health Sciences, Makerere University, P.O. Box 7072, Kampala, Uganda \\ ${ }^{3}$ Mulago National Referral \& Teaching Hospital, P.O. Box 7051, Kampala, Uganda \\ ${ }^{4}$ Faculty of Health Sciences, Busitema University, P.O. Box 1460, Mbale, Uganda
}

Correspondence should be addressed to Milton W. Musaba; miltonmusaba@gmail.com

Received 16 November 2016; Revised 9 January 2017; Accepted 18 January 2017; Published 9 February 2017

Academic Editor: David Baker

Copyright (C) 2017 Milton W. Musaba et al. This is an open access article distributed under the Creative Commons Attribution License, which permits unrestricted use, distribution, and reproduction in any medium, provided the original work is properly cited.

\begin{abstract}
Background. A 2013 Cochrane review concluded that the choice of antibiotics for prophylaxis in PROM is not clear. In Uganda, a combination of oral erythromycin and amoxicillin is the 1st line for prophylaxis against ascending infection. Our aim was to establish the current cervicovaginal bacteriology and antibiotic sensitivity patterns. Methods. Liquor was collected aseptically from the endocervical canal and pool in the posterior fornix of the vagina using a pipette. Aerobic cultures were performed on blood, chocolate, and MacConkey agar and incubated at $35-37^{\circ} \mathrm{C}$ for $24-48 \mathrm{hrs}$. Enrichment media were utilized to culture for GBS and facultative anaerobes. Isolates were identified using colonial morphology, gram staining, and biochemical analysis. Sensitivity testing was performed via Kirby-Bauer disk diffusion and dilution method. Pearson's chi-squared $\left(\chi^{2}\right)$ test and the paired $t$-test were applied, at a $P$ value of 0.05 . Results. Thirty percent of the cultures were positive and over $90 \%$ were aerobic microorganisms. Resistance to erythromycin, ampicillin, cotrimoxazole, and ceftriaxone was 44\%, $95 \%, 96 \%$, and $24 \%$, respectively. Rupture of membranes $(>12 \mathrm{hrs})$, late preterm, and term PROM were associated with more positive cultures. Conclusion. The spectrum of bacteria associated with PROM has not changed, but resistance to erythromycin and ampicillin has increased.
\end{abstract}

\section{Background}

Prelabour rupture of membranes is the commonest antecedent to preterm and term labour [1]. The aetiology of preterm delivery has been attributed to a number of causes such as decidual hemorrhage, inflammation of the fetal membranes, activation of the maternal-fetal hypothalamic pituitary axis, structural abnormalities like cervical incompetence, pathologic distension of the uterus, and uterine abnormalities [2]. However, there is strong epidemiological and biochemical evidence which links preterm and term delivery to ascending infection of the female genital tract [3].

The incidence of maternal and neonatal sepsis ranges from 1 to $25 \%$ depending on the duration of rupture of membranes and gestation age [4] or even higher in low resource settings [5]. In Uganda, $22 \%$ of the maternal deaths are due to sepsis [6] and sepsis is the 2nd major cause of neonatal mortality [7]. Premature rupture of membranes at or near term complicates 3\% of all pregnancies in Mulago Hospital [8]. Anecdotal evidence suggests that most of these patients suffer adverse obstetric outcomes due to sepsis.

Several organisms have been variably associated with PROM in different parts of the world [1]. Previous culture and sensitivity studies in this settings have involved sick neonates and patients with surgical site infections $[9,10]$. Only one found that $13.3 \%$ of 180 women with PROM in Mulago Hospital had positive amniotic fluid cultures with descending order isolates of Escherichia coli, beta hemolytic Streptococcus, 
Proteus species, and Peptostreptococcus, although the antibiotic sensitivity patterns were not done [8].

The choice of antibiotics for prophylaxis should be based on culture and sensitivity patterns as well as epidemiological patterns, but because of limited access to reliable laboratory services in Uganda, broad spectrum antibiotics are given empirically. A combination of oral erythromycin $(250 \mathrm{mg}$ ) and amoxicillin $(500 \mathrm{mg}$ ) for 7 days is the 1st line for prophylaxis against ascending genital tract infection following PROM (MOH 2012). It is not clear if this recommendation is based on current culture and sensitivity patterns. In Africa, resistance to commonly used antibiotics has greatly increased over the last 20 years [9]; so we are no longer sure if this recommendation is still relevant. A recent Cochrane review which included 22 studies from high resource settings concluded that the antibiotic of choice for prophylaxis in PROM is not clear [11]. Probably the associated organisms have changed or developed resistance, hence the need to establish the current bacterial culture and sensitivity patterns in this population of patients. This information may inform our choice of antibiotics for prophylaxis against ascending infection following PROM and probably contribute to the body of evidence needed to either revise or maintain the current guideline for management of PROM.

The aim of this study was to establish the current cervicovaginal bacteriology and antibiotic sensitivity patterns.

\section{Materials and Methods}

2.1. Study Design. A cross-sectional study was conducted on the labour suite at Mulago National Referral and Teaching Hospital Uganda between January and May, 2013.

2.2. Study Setting. Mulago Hospital is the national referral hospital for Uganda and a teaching hospital for Makerere University. The hospital averages 30,000 births per year. In 2011, 868 patients with PROM were admitted in Mulago Hospital, none of them had culture and sensitivity patterns done and the pregnancy outcomes related to sepsis were not easy to ascertain because of poor documentation.

2.3. Sample Size Calculation. The Leslie Kish (1965) formula for determining single proportions was used. $P$ was the percentage of positive amniotic fluid cultures among patients with PROM in Mulago National hospital, using 13.3\% [8].

2.4. Sampling. We consecutively enrolled 196 women with a diagnosis of PROM and a viable foetus at or after 28 weeks of gestation, over a 5 months' period. Ethical approval was obtained from the School of Medicine Research and Ethics Committee, Makerere University College of Health Sciences, and the Uganda National Council for Science and Technology. Each participant was requested to give written informed consent. An interviewer administered questionnaire was used to collect data on patient demographics and clinical features [12].

Using an aseptic technique, $5 \mathrm{mLs}$ of amniotic fluid was collected from both the endocervical canal and the pool in the posterior fornix of the vagina using a pipette
TABLE 1: Sociodemographic and clinical characteristics $(n=196)$.

\begin{tabular}{lc}
\hline Characteristic & Frequency $(\%)$ \\
\hline Mean age & $25(16-47)$ years \\
Referral status & \\
$\quad$ Yes & $54(27.6)$ \\
No & $142(72.4)$ \\
Gestation age & \\
$\quad$ Extreme preterm (28-31 weeks) & $19(10)$ \\
Late preterm (32-36 weeks) & $52(27)$ \\
Term $(>37$ weeks) & $125(64)$ \\
Gravidity & \\
$\quad$ Primigravida & $80(40)$ \\
$2-3$ & $60(31)$ \\
$>3$ & $56(29)$ \\
Prior antibiotic use & \\
Yes & $46(23.5)$ \\
No & $150(76.5)$ \\
Duration of ROM & \\
$<12$ hrs & $77(39)$ \\
$12-24$ hrs & $20(10)$ \\
$>24$ hrs & $99(51)$ \\
\hline
\end{tabular}

TABLE 2: Isolates from the positive cervicovaginal cultures.

\begin{tabular}{|c|c|}
\hline & Number \\
\hline \multicolumn{2}{|l|}{ Gram negative organism } \\
\hline Bacteroides species* & 3 \\
\hline Citrobacterfreundii** & 2 \\
\hline Enterobacterspecies & 1 \\
\hline Escherichiacoli & 12 \\
\hline KlebsiellaPneumoniae & 7 \\
\hline \multicolumn{2}{|l|}{ Gram positive organisms } \\
\hline Clostridiumtetani $^{*}$ & 1 \\
\hline Coagulase-negative Staphylococcus & 9 \\
\hline Coryneform bacteria & 1 \\
\hline Enterococcus species & 3 \\
\hline Staphylococcus aureus & 13 \\
\hline Streptococcus pyogenes & 8 \\
\hline Streptococcuspneumoniae & 1 \\
\hline Streptococcusspecies & 1 \\
\hline Viridiansstreptococcus ${ }^{* *}$ & 3 \\
\hline Listeriamonocytogenes & 1 \\
\hline Positive rods isolated ${ }^{*}$ & 1 \\
\hline Total & 67 \\
\hline
\end{tabular}

${ }^{*}$ Anaerobe. ${ }^{* *}$ Facultative anaerobe.

and transported to the microbiology laboratory within 30 minutes for analysis. Specimen was transported in 3 separate containers: $1 \mathrm{~mL}$ inoculated into a selective Todd-Hewitt enrichment media to culture for GBS in a glass bottle; $1 \mathrm{~mL}$ inoculated into thioglycolate enrichment media to culture for facultative anaerobes in a glass bottle; $3 \mathrm{~mL}$ in a sterile plastic container. The aerobic culture was performed on blood, 
TABLE 3: Antibiotic sensitivity profile of positive cervicovaginal cultures.

\begin{tabular}{|c|c|c|c|c|}
\hline Antibiotic & Sensitive (\%) & Resistant (\%) & Intermediate (\%) & Total \\
\hline Ceftriaxone/cefotaxime & $16(76)$ & $5(24)$ & 0 & 21 \\
\hline Cotrimoxazole & $1(4)$ & $26(96)$ & 0 & 27 \\
\hline Erythromycin & $15(56)$ & $10(37)$ & $2(7)$ & 27 \\
\hline Vancomycin & $20(95)$ & $1(5)$ & 0 & 21 \\
\hline Chloramphenicol & $23(62)$ & $14(38)$ & 0 & 37 \\
\hline Clindamycin & $6(40)$ & $7(47)$ & $2(13)$ & 15 \\
\hline Ampicillin & $1(5)$ & $20(95)$ & 0 & 21 \\
\hline Penicillin G & $9(38)$ & $15(63)$ & 0 & 24 \\
\hline Co-amoxiclav & $3(17)$ & $15(83)$ & 0 & 18 \\
\hline Tetracycline & $6(21)$ & $22(79)$ & 0 & 28 \\
\hline Meropenem & $6(100)$ & 0 & 0 & 6 \\
\hline Ciprofloxacin & $21(66)$ & $11(34)$ & 0 & 32 \\
\hline Oxacillin & $1(7)$ & $14(93)$ & 0 & 15 \\
\hline Ofloxacin & $4(80)$ & $1(20)$ & 0 & 5 \\
\hline Cefazolin & $3(23)$ & $9(69)$ & $1(8)$ & 13 \\
\hline Cefuroxime & $3(19)$ & $13(81)$ & 0 & 16 \\
\hline Gentamicin & $12(46)$ & $12(46)$ & $2(8)$ & 26 \\
\hline Ceftazidime & $2(100)$ & 0 & 0 & 2 \\
\hline Nitrofurantoin & $1(100)$ & 0 & 0 & 1 \\
\hline Cefotaxime-clavulanic acid & $1(100)$ & 0 & 0 & 1 \\
\hline
\end{tabular}

chocolate, and MacConkey agar incubated at 35-37 for 2448 hours [12]. Colonial morphology, gram staining, and biochemical analysis were used to identify the isolates. The Kirby-Bauer disk diffusion and dilution methods were used to test for sensitivity of the isolates against nitroimidazoles, penicillins, cephalosporins, sulfonamides, aminoglycosides, quinolones/fluoroquinolones, macrolides, and tetracyclines, as specified in the manual of clinical microbiology (Jorgensen, Pfaller et al. 2015). Ten percent of the samples were taken to 2 laboratories (Medical School Laboratory and MBN diagnostic laboratories) for quality control purposes [12].

Data was entered into Epidata version 3.1 and then exported into Stata version 12 for analysis. The proportion of positive amniotic fluid cultures was computed; statistical significance was tested via the chi $\left(\chi^{2}\right)$ test [12]. The paired $t$-test was applied to measure the significance of the factors associated with positive cultures $(P=0.05)$.

\section{Results}

Fifty-eight (30\%) of the 196 cervicovaginal cultures were positive. A quarter of the participants had received an antibiotic prior to admission and the most commonly used antibiotics were ceftriaxone (29), ampicillin (13), erythromycin (9), and metronidazole (9) (Table 1).

Sixty-seven isolates were identified from the 58 positive cultures, $63 \%$ were gram positive aerobic bacteria, and only 5 (7.5\%) were anaerobes (Table 2 ).

Resistance to most commonly used broad spectrum antibiotics (ceftriaxone, ampicillin, cotrimoxazole, and erythromycin) was notably high. It is only the less commonly used and more expensive antibiotics like vancomycin and meropenem that showed the highest levels of sensitivity to the isolated antibiotics (Table 3).

Rupture of membranes for $>12$ hrs and late preterm and term PROM mothers were associated with a higher percentage of positive cultures. On the other hand prior use of any antibiotic following rupture of membranes and a positive HIV status were associated with fewer positive cultures (Table 4).

\section{Discussion}

In the current study, we found that the proportion of positive cervicovaginal cultures has doubled since 1996 [8]. This could be attributed to the use of enrichment broths to increase the yield of specific bacterial isolates. A review of 18 published studies involving 1,727 women with PROM in which amniotic fluid was obtained by amniocentesis; a $32.4 \%$ rate of positive cultures was reported for women not in labour and $75 \%$ for those admitted in labour [13]. Despite the differences in setting and techniques employed, the rate of positive cultures is similar.

The spectrum of bacteria associated with PROM has not changed much over time [8] and it is similar to those isolated from neonates with sepsis in this hospital [7].

We did not isolate any GBS despite the fact that an enrichment medium was used to favour its growth. It has been strongly associated with neonatal sepsis following vertical transmission. This could be so because either we used an antiseptic before introducing a speculum into the vagina or the site, or timing and nature of specimen collected were not ideal [14] 
TABLE 4: Factors associated with cervicovaginal culture and sensitivity patterns.

\begin{tabular}{|c|c|c|c|c|}
\hline Characteristic & Positive cultures & Negative cultures & Odds ratio $(\mathrm{CI})$ & $P$ values \\
\hline \multicolumn{5}{|l|}{ Referral status } \\
\hline Yes & $14(26 \%)$ & $40(74 \%)$ & \multirow{2}{*}{0.78 (CI 0.49-1.38) } & \multirow{5}{*}{0.488} \\
\hline No & $44(31 \%)$ & $98(69 \%)$ & & \\
\hline Mean maternal BP & $119 / 74$ & $116 / 75$ & Mean $=117 / 75$ & \\
\hline Mean radial pulse & 83 & 85 & Mean $=85$ & \\
\hline Mean axillary temp. & 37 & 37 & Mean $=37$ & \\
\hline \multicolumn{5}{|l|}{ Gestation age (weeks) } \\
\hline Extreme preterm (28-31) & $8(42 \%)$ & $11(58 \%)$ & \multirow{3}{*}{$1.4(\mathrm{CI} 1.3-1.6)$} & \multirow{3}{*}{0.000} \\
\hline Late preterm $(32-36)$ & $16(31 \%)$ & $36(39 \%)$ & & \\
\hline Term $(>37)$ & $34(27 \%)$ & $91(73 \%)$ & & \\
\hline \multicolumn{5}{|l|}{ Gravidity } \\
\hline Primigravida & $24(30 \%)$ & $56(70 \%)$ & \multirow{3}{*}{1.12 (CI 0.77-1.62) } & \multirow{3}{*}{0.343} \\
\hline $2-3$ & $14(23 \%)$ & $46(77 \%)$ & & \\
\hline$>3$ & $20(36 \%)$ & $36(64 \%)$ & & \\
\hline \multicolumn{5}{|l|}{ Duration of ROM } \\
\hline$<12$ & $16(21 \%)$ & $61(79 \%)$ & \multirow{3}{*}{1.33 (CI 0.96-1.85) } & \multirow{3}{*}{0.055} \\
\hline $12-24$ & $9(45 \%)$ & $11(55 \%)$ & & \\
\hline$>24$ & $33(33 \%)$ & $66(67 \%)$ & & \\
\hline \multicolumn{5}{|l|}{ HIV serology } \\
\hline Negative & $50(29 \%)$ & $123(71 \%)$ & \multirow{2}{*}{0.76 (CI 0.30-1.92) } & \multirow{2}{*}{0.562} \\
\hline Positive & $8(35 \%)$ & $15(65 \%)$ & & \\
\hline \multicolumn{5}{|l|}{ Prior antibiotic use } \\
\hline Yes & $9(20 \%)$ & $37(80 \%)$ & \multirow{2}{*}{0.50 (CI 0.22-1.13) } & \multirow{2}{*}{0.089} \\
\hline No & $49(33 \%)$ & $101(67 \%)$ & & \\
\hline \multicolumn{5}{|l|}{ Prior steroid use } \\
\hline Yes & $7(41 \%)$ & $10(59 \%)$ & \multirow{2}{*}{1.76 (CI 0.63-4.90) } & \multirow{2}{*}{0.274} \\
\hline No & $51(28 \%)$ & $128(72 \%)$ & & \\
\hline \multicolumn{5}{|l|}{ Regular medication use (ART) } \\
\hline Yes & $6(35 \%)$ & $11(65 \%)$ & \multirow{2}{*}{1.33 (CI 0.47-3.80) } & \multirow{2}{*}{0.59} \\
\hline No & $52(29 \%)$ & $127(71 \%)$ & & \\
\hline \multicolumn{5}{|l|}{ Mean FHR } \\
\hline$<120$ & $0(0 \%)$ & $2(100 \%)$ & \multirow{3}{*}{1.30 (CI 0.37-4.54) } & \multirow{3}{*}{0.654} \\
\hline $120-160$ & $55(30 \%)$ & $129(70 \%)$ & & \\
\hline$>160$ & $3(30 \%)$ & $7(70 \%)$ & & \\
\hline Gram stain result & & & & \\
\hline Positive & $38(50 \%)$ & $38(50 \%)$ & $0.2($ CI $0.10-0.40)$ & $<0.001$ \\
\hline Negative & $20(17 \%)$ & $100(83 \%)$ & & \\
\hline
\end{tabular}

The yield of anaerobic bacteria was very poor, even though specific measures like the use of enrichment media for transportation of cervicovaginal samples were employed in this study. Several studies from high income settings have strongly associated them with PROM [4], so it would be interesting to study the whole picture in a better resourced laboratory before drawing conclusions [12].

Resistance to commonly used antibiotics is high which is consistent with reports of increasing antibiotic resistance in resource limited setting due to irrational use of antibiotics [9]. Due to limitations in accessing laboratory services in Uganda and other similar settings, there is a recourse to empirical use of broad spectrum antibiotics for prophylaxis against ascending genital tract infections following PROM [12]. The findings of high resistance to erythromycin and ampicillin, the 2 antibiotics recommended by $\mathrm{MOH}$ in Uganda, raise concerns regarding their usefulness as 1st choice prophylactic antibiotics. It is possible that either the organisms have developed resistance over time or the associated organisms have changed. Moving forward, trials need to be undertaken to identify the most appropriate antibiotic for prophylaxis following PROM in Uganda.

Although this study was not powered to determine associated factors, it was noted that there were more positive cultures when the duration of rupture of membranes was longer than 24 hrs but this result was not statistically significant; 
TABLE 5: Gestation age and cervicovaginal cultures of mothers with PROM.

\begin{tabular}{lccr}
\hline & Odds ratio & $P$ value & \\
\hline Extreme preterm $(28-31) \mathrm{R}$ & 1.0 & & \\
Late preterm $(32-36)$ versus extreme preterm $(28-31)$ & 2.3 & 0.007 & 1.2 \\
Term $(>37)$ versus extreme preterm $(28-31)$ & 2.7 & 0.000 & 4.1 \\
\hline
\end{tabular}

$P=0.055$. It is also important to note that as early as $12 \mathrm{hrs}$ after ROM $21 \%$ of 77 cultures were positive (Table 4 ). In this setting, we probably need to initiate prophylaxis early even before the recommended $18 \mathrm{hrs}$ for prolonged ROM. A similar trend was also noted with the gestation age; there were more positive cultures seen with decreasing gestation age. This is consistent with other studies that have identified these two factors as important risk factors for ascending genital tract infection following PROM [15]. Further analysis revealed that mothers with late preterm and term PROM were more likely to have positive cultures compared to mothers with extreme preterm PROM. Both of them were statistically significant $P=0.007$ and $P=0.000$, respectively (Table 5).

Prior use of antibiotics following PROM was associated with fewer positive cervicovaginal cultures, which is expected. Although in this current study, only $23.5 \%$ of the participants reported use of antibiotics prior to admission following PROM.

\section{Conclusions}

The proportion of positive cervicovaginal cultures in Mulago National Referral and Teaching Hospital has more than doubled, but the spectrum of associated bacteria isolated has not changed since 1996. Resistance to the most commonly used antibiotics in our setting is high. There is a need for clinical trials to identify the most appropriate antibiotic for prophylaxis in PROM.

\section{Abbreviations}

PROM: Premature rupture of membranes

C \& S: Culture and sensitivity patterns

GBS: Group B Streptococcus

$\mathrm{MOH}$ : Ministry of Health.

\section{Competing Interests}

The authors declare that they have no competing interests.

\section{Authors' Contributions}

Milton W. Musaba participated in conception and design of the proposal, collection of the data and analysis, and drafting the manuscript. Julius Wandabwa and Paul Kiondo were involved in drafting and reviewing the manuscript for accuracy and intellectual content. Mike N. Kagawa and Charles Kiggundu made substantial contribution to the conception and design of the proposal as well as interpretation of the data. All the author's read and approved the final manuscript.

\section{Acknowledgments}

Thanks are due to the Staff of Medical School Microbiology Laboratory and MBN Clinical Laboratories for working tirelessly and Javis Tumuhe for helping with the statistical work.

\section{References}

[1] S. L. Kenyon, D. J. Taylor, and W. Tarnow-Mordi, "Broadspectrum antibiotics for preterm, prelabour rupture of fetal membranes: the ORACLE I randomised trial," Lancet, vol. 357, no. 9261, pp. 979-988, 2001.

[2] E. R. Newton, "Preterm labor, preterm premature rupture of membranes, and chorioamnionitis," Clinics in Perinatology, vol. 32, no. 3, pp. 571-600, 2005.

[3] G. G. G. Donders and G. Bellen, "Management of abnormal vaginal flora as a risk factor for preterm birth," in Preterm Birth-Mother and Child, InTech, 2011.

[4] A. T. N. Tita and W. W. Andrews, "Diagnosis and management of clinical chorioamnionitis," Clinics in Perinatology, vol. 37, no. 2, pp. 339-354, 2010.

[5] A. C. Seale, M. Mwaniki, C. R. Newton, and J. A. Berkley, "Maternal and early onset neonatal bacterial sepsis: burden and strategies for prevention in sub-Saharan Africa," The Lancet Infectious Diseases, vol. 9, no. 7, pp. 428-438, 2009.

[6] $\mathrm{MOH}$, Uganda Clinical Guidelines, 2012.

[7] J. Mugalu, M. K. Nakakeeto, S. Kiguli, and D. H. KadduMulindwa, "Aetiology, risk factors and immediate outcome of bacteriologically confirmed neonatal septicaemia in Mulago hospital, Uganda," African Health Sciences, vol. 6, no. 2, pp. 120 126, 2006.

[8] D. Kaye, "Risk factors for preterm premature rupture of membranes at Mulago Hospital, Kampala," East African Medical Journal, vol. 78, no. 2, pp. 65-69, 2001.

[9] A. N. Kimang'a, "A situational analysis of antimicrobial drug resistance in Africa: are we losing the battle?" Ethiopian Journal of Health Sciences, Jimma, Ethiopia, vol. 22, pp. 135-143, 2012.

[10] Y. Mpairwe and S. Wamala, Antibiotic Resistance in Uganda: Situation Analysis and Recommendations, Uganda National Academy of Sciences, Kampala, Uganda, 2015.

[11] S. Kenyon, M. Boulvain, and J. Neilson, "Antibiotics for preterm rupture of membranes (Review)," The Cochrane Database of Systematic Reviews, no. 12, Article ID CD001058, 2010.

[12] L. Mubangizi, F. Namusoke, and T. Mutyaba, "Aerobic cervical bacteriology and antibiotic sensitivity patterns in patients with advanced cervical cancer before and after radiotherapy at a national referral hospital in Uganda," International Journal of Gynecology and Obstetrics, vol. 126, no. 1, pp. 37-40, 2014.

[13] R. Romero, R. Gómez, T. Chaiworapongsa, G. Conoscenti, J. C. Kim, and Y. M. Kim, "The role of infection in preterm labour and delivery," Paediatric and Perinatal Epidemiology, vol. 15, no. 2, pp. 41-56, 2001. 
[14] A. Joachim, M. I. Matee, F. A. Massawe, and E. F. Lyamuya, "Maternal and neonatal colonisation of group B streptococcus at Muhimbili National Hospital in Dar es Salaam, Tanzania: prevalence, risk factors and antimicrobial resistance," $B M C$ Public Health, vol. 9, article no. 437, 2009.

[15] M. E. Hannah, A. Ohlsson, D. Farine et al., "Induction of labor compared with expectant management for prelabor rupture of the membranes at term," New England Journal of Medicine, vol. 334 , no. 16, pp. 1005-1010, 1996. 


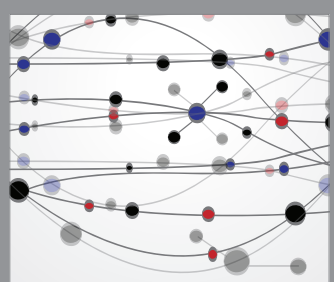

The Scientific World Journal
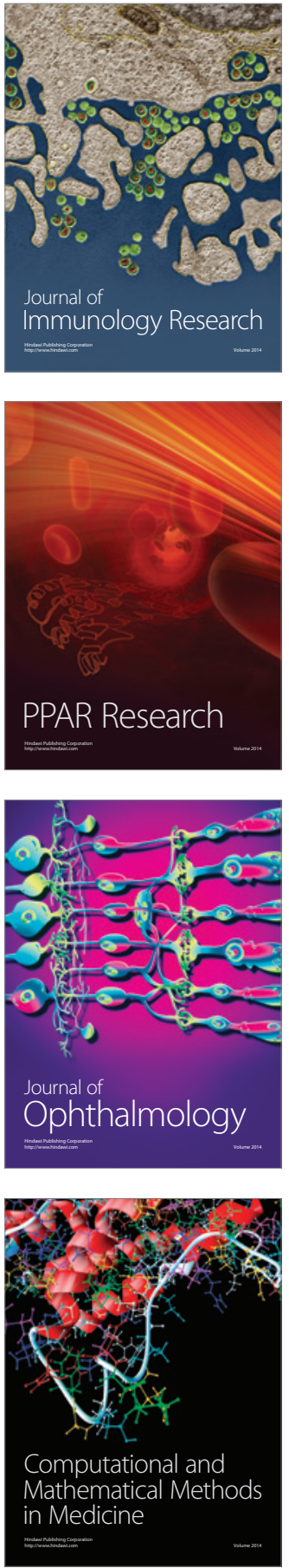

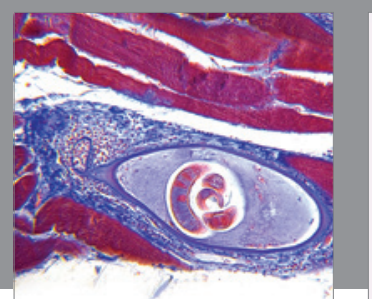

Gastroenterology Research and Practice
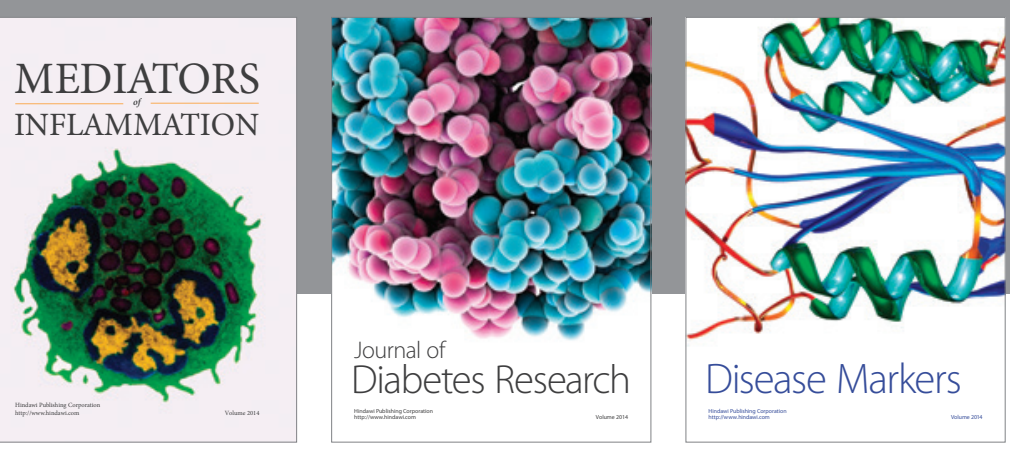

Disease Markers

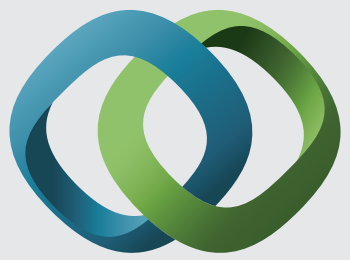

\section{Hindawi}

Submit your manuscripts at

https://www.hindawi.com
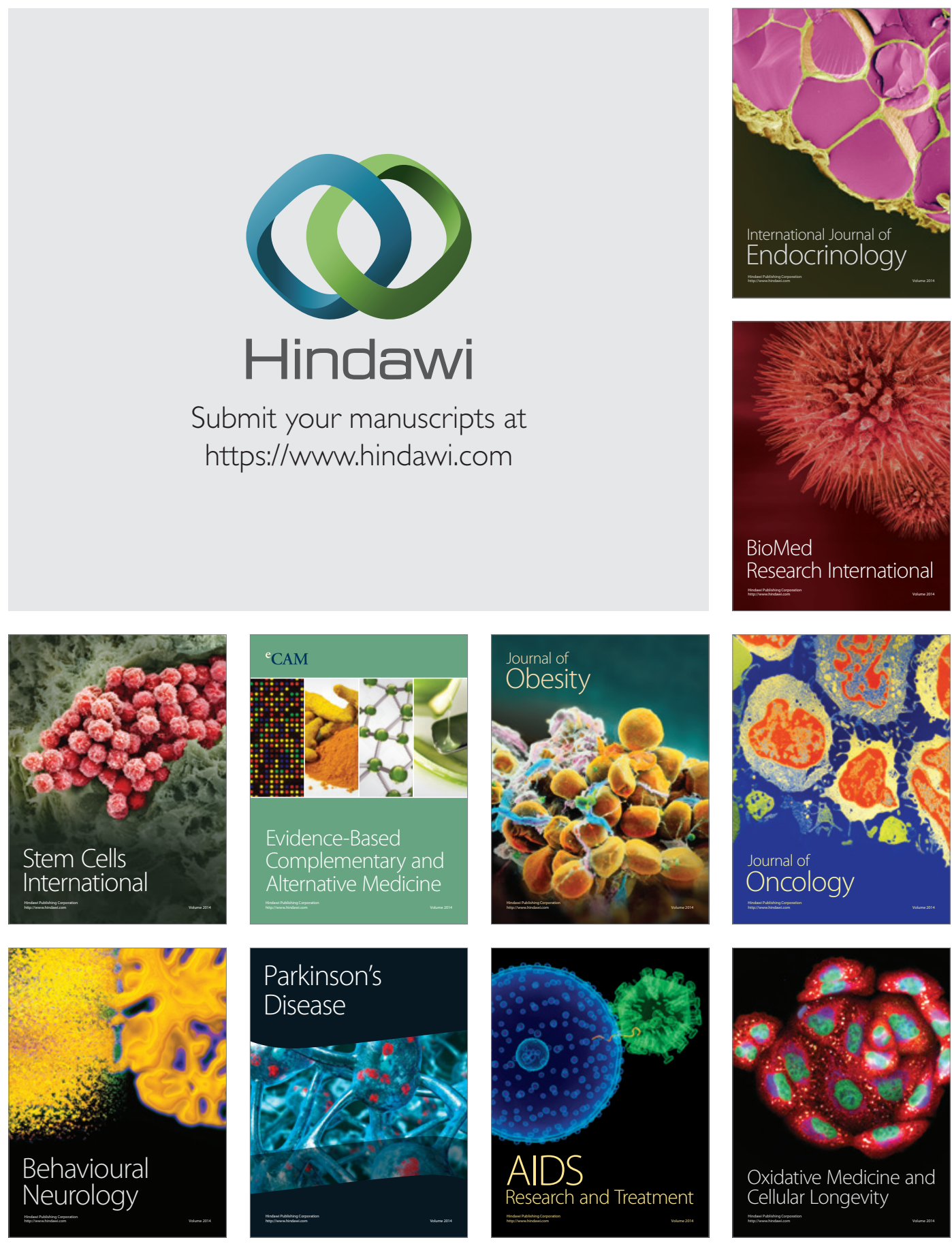